\title{
The role of diffusion tensor imaging and fractional anisotropy in the evaluation of patients with idiopathic normal pressure hydrocephalus: a literature review
}

\author{
Ioannis Siasios, MD, MSc, ${ }^{1,2}$ Eftychia Z. Kapsalaki, MD, PhD, ${ }^{3}$ Kostas N. Fountas, MD, PhD, ${ }^{4}$ \\ Aggeliki Fotiadou, MS, ${ }^{5}$ Alexander Dorsch, MD, ${ }^{1,2}$ Kunal Vakharia, MD, ${ }^{1,2}$ John Pollina, MD, ${ }^{1,2}$ and \\ Vassilios Dimopoulos, MD ${ }^{1,2}$
}

'Department of Neurosurgery, Jacobs School of Medicine and Biomedical Sciences, University at Buffalo, State University of New York; ${ }^{2}$ Department of Neurosurgery, Kaleida Health, Buffalo, New York; and Departments of ${ }^{3}$ Diagnostic Radiology, ${ }^{4}$ Neurosurgery, and ${ }^{5}$ Medicine, University of Thessaly, Larisa, Greece

OBJECTIVE Diffusion tensor imaging (DTI) for the assessment of fractional anisotropy (FA) and involving measurements of mean diffusivity (MD) and apparent diffusion coefficient (ADC) represents a novel, MRI-based, noninvasive technique that may delineate microstructural changes in cerebral white matter (WM). For example, DTI may be used for the diagnosis and differentiation of idiopathic normal pressure hydrocephalus (iNPH) from other neurodegenerative diseases with similar imaging findings and clinical symptoms and signs. The goal of the current study was to identify and analyze recently published series on the use of DTI as a diagnostic tool. Moreover, the authors also explored the utility of DTI in identifying patients with iNPH who could be managed by surgical intervention.

METHODS The authors performed a literature search of the PubMed database by using any possible combinations of the following terms: "Alzheimer's disease," "brain," "cerebrospinal fluid," "CSF," "diffusion tensor imaging," "DTI," "hydrocephalus," "idiopathic," "magnetic resonance imaging," "normal pressure," "Parkinson's disease," and "shunting." Moreover, all reference lists from the retrieved articles were reviewed to identify any additional pertinent articles.

RESULTS The literature search retrieved 19 studies in which DTI was used for the identification and differentiation of iNPH from other neurodegenerative diseases. The DTI protocols involved different approaches, such as region of interest (ROI) methods, tract-based spatial statistics, voxel-based analysis, and delta-ADC analysis. The most studied anatomical regions were the periventricular WM areas, such as the internal capsule (IC), the corticospinal tract (CST), and the corpus callosum (CC). Patients with iNPH had significantly higher MD in the periventricular WM areas of the CST and the CC than had healthy controls. In addition, FA and ADCs were significantly higher in the CST of iNPH patients than in any other patients with other neurodegenerative diseases. Gait abnormalities of iNPH patients were statistically significantly and negatively correlated with FA in the CST and the minor forceps. Fractional anisotropy had a sensitivity of $94 \%$ and a specificity of $80 \%$ for diagnosing iNPH. Furthermore, FA and MD values in the CST, the IC, the anterior thalamic region, the fornix, and the hippocampus regions could help differentiate iNPH from Alzheimer or Parkinson disease. Interestingly, CSF drainage or ventriculoperitoneal shunting significantly modified FA and ADCs in iNPH patients whose condition clinically responded to these maneuvers.

CONCLUSIONS Measurements of FA and MD significantly contribute to the detection of axonal loss and gliosis in the periventricular WM areas in patients with iNPH. Diffusion tensor imaging may also represent a valuable noninvasive method for differentiating iNPH from other neurodegenerative diseases. Moreover, DTI can detect dynamic changes in the WM tracts after lumbar drainage or shunting procedures and could help identify iNPH patients who may benefit from surgical intervention.

http://thejns.org/doi/abs/10.3171/2016.6.FOCUS16192

KEY WORDS Alzheimer disease; diffusion tensor imaging; fractional anisotropy; idiopathic; normal pressure hydrocephalus; Parkinson disease; vascular disorders

ABBREVIATIONS AD = Alzheimer disease; $A D C=$ apparent diffusion coefficient; $C C=$ corpus callosum; $C S T=$ corticospinal tract; $D K I=$ diffusional kurtosis imaging; $D T I=$ diffusion tensor imaging; FA = fractional anisotropy; IC = internal capsule; $\mathrm{NPH}=$ idiopathic NPH; $\mathrm{MD}=$ mean diffusivity; $\mathrm{MTR}=$ magnetization transfer ratio; NPH = normal pressure hydrocephalus; $\mathrm{PD}=$ Parkinson disease; $\mathrm{ROI}=$ region of interest; $\mathrm{TBSS}=$ tract-based spatial statistics; $\mathrm{WM}=$ white matter .

SUBMITTED May 1, 2016. ACCEPTED June 27, 2016.

INCLUDE WHEN CITING DOI: 10.3171/2016.6.FOCUS16192. 
$\mathrm{N}$ ORMAL pressure hydrocephalus (NPH), first described in 1965 by Salomon Hakim, is defined as a clinicopathological condition related to the overproduction or flow obstruction of CSF. ${ }^{13,39}$ The prevalence of this disease is age related, reaching 1\%-5.9\% among elderly individuals. ${ }^{21,26}$ It remains one of the most controversial neuropathological entities both in regards to its diagnosis and its proper management. Characteristically, despite many recent advances in imaging methods, most NPH cases still remain idiopathic. Normal pressure hydrocephalus is usually characterized as idiopathic, although it can be secondarily present in several disorders and conditions such as subarachnoid hemorrhage, head trauma, and CNS tumors. Patients with idiopathic NPH (iNPH) have enlarged ventricles but normal CSF pressure seen at a diagnostic lumbar puncture. ${ }^{1}$ The most common symptoms of iNPH are summarized in a clinical triad: gait disturbance, dementia, and urinary incontinence. ${ }^{58}$ However, these symptoms are not pathognomonic and may also be present in vascular dementia, Alzheimer disease (AD), and Parkinson disease (PD), among many other less common diseases. The exact underlying pathophysiological mechanism of this disorder has remained ill defined, and several theories supporting the combination of CSF circulation disturbances along with other cerebrovascular disorders have been proposed. $1,21,28,36,41,58$

The diagnosis of NPH is based on a patient's clinical status, imaging findings, and the natural history of the disease. The imaging criteria for NPH are mainly ventricular enlargement (i.e., with an Evans index >0.3), lack of any anatomical obstruction of the CSF circulation, enlarged temporal horns, and a corpus callosum (CC) angle of greater than $40^{\circ}$ on brain CT or MRI scans. ${ }^{58}$ Other diagnostic methods are a radionuclide cisternogram showing delayed clearance (i.e., of 48-72 hours) of the infused radiotracer over the cerebral convexities, a brain cine MRI study revealing increased ventricular flow rate, and a SPECT scan indicating decreased periventricular perfusion. ${ }^{25,31,57}$

Recently, several clinical investigators have employed diffusion tensor imaging (DTI) techniques to detect and quantify any WM alterations in patients with iNPH. The DTI data generally indicate the movements of water molecules inside the brain tissue. The combination of 2D diffusion-weighted images with all diagonal elements in a 3D diffusion model creates a high-resolution MRI series, which may identify even subtle structural changes in the periventricular white matter (WM). Quantitative measurements in these changes are enabled by fractional anisotropy (FA) and apparent diffusion coefficient (ADC) methods, which can be used to determine the structural integrity of the brain tissue. ${ }^{4}$ Researchers have used 3 basic models to analyze DTI data: region of interest (ROI) analysis, tract-specific analysis, and voxel-wise statistical analysis. The ROI analysis has the disadvantage of subjectivity. The tract-specific analysis is applied only to regions of the brain in which diffusion can be accurately measured. Voxel-wise analysis is more useful in measuring WM changes, since it can be performed on the whole brain. The iNPH-associated axonal loss could explain the observed decreased FA in the anterior frontal WM, a find- ing that is also confirmed by proton MR spectroscopy and magnetization transfer studies. ${ }^{19,35}$

In the current study, we reviewed and analyzed the literature data on the use of MRI-based DTI in the differential diagnosis of iNPH from other neurodegenerative diseases, such as PD or AD, with similar clinical symptoms and conventional MRI findings. Moreover, the potential prognostic role of DTI for outcomes among iNPH patients undergoing surgical CSF shunt placement was evaluated.

\section{Methods}

An extensive literature search in the PubMed database was performed for the period of January 2010 to December 2015. The following terms were used in this search: "Alzheimer's disease," "brain," "cerebrospinal fluid," "CSF," "diffusion tensor imaging," "DTI," "hydrocephalus," "idiopathic," "magnetic resonance imaging," "normal pressure," "Parkinson's disease," and "shunting," in all possible combinations. The search was limited to articles published in English, and case reports were excluded.

All retrieved articles were separately reviewed by 2 of the authors (A.F. and I.S.). In addition, the reference lists of all the retrieved articles were meticulously reviewed to identify any additional pertinent articles. In the review of the articles identified, particular attention was paid to the study design, its methodological characteristics, the technical characteristics of the FA and DTI protocols used, and the potential clinical significance of the DTI and FA findings. Of note, we made every possible effort to identify any redundant data among the different articles.

\section{Results}

Our literature search identified 19 studies that are included in the current review (Table 1). The retrieved studies were separated, whenever possible, into 3 groups: 1) studies attempting to define the FA and DTI characteristics in iNPH patients by comparison with the corresponding characteristics in age-matched, healthy study participants; 2) studies examining the role of FA and DTI in the accurate diagnosis of iNPH and particularly in differentiating iNPH from other neurodegenerative diseases with similar signs and symptoms; and 3) studies evaluating the potential prognostic role of FA and DTI for iNPH patients undergoing CSF drainage or surgical shunt placement. In the following, we detail the findings in these selected studies.

\section{DTI Characteristics in iNPH Patients and Aged-Matched Healthy Controls}

Hattingen et al. compared the DTI characteristics in 11 patients with possible iNPH with those in 10 healthy controls. ${ }^{14}$ All study participants underwent 3T brain MRI in which 12 DTI gradient directions were acquired. The authors compared FA and mean diffusivity (MD) between patients and controls either by using manual definition of ROIs or by employing a fully automated definition of ROI with tract-based spatial statistics (TBSS). Compared with the healthy controls, the patients with suspected iNPH had 


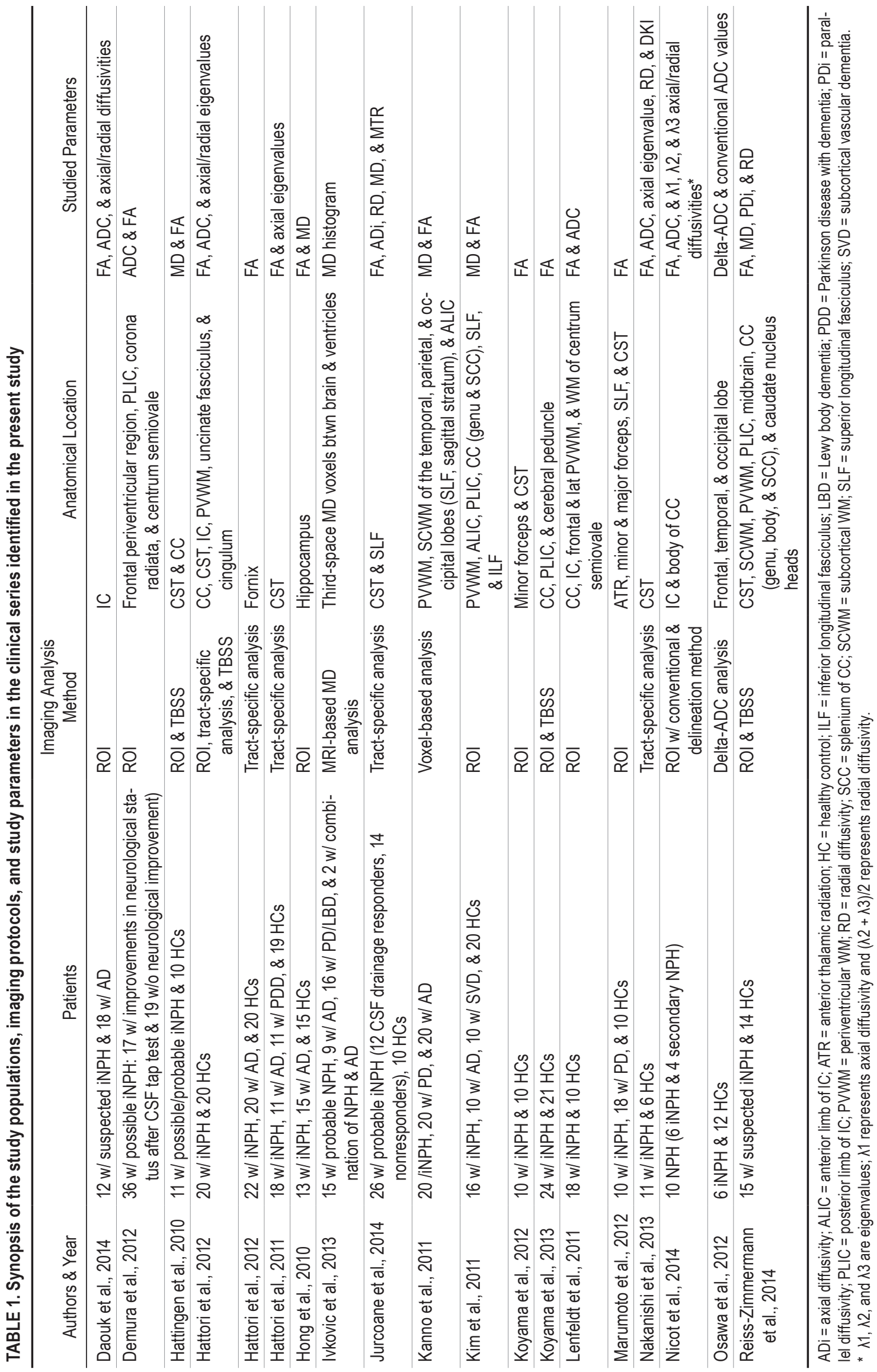


statistically significantly increased MD in the periventricular corticospinal tract (CST) and the CC. Fractional anisotropy was also significantly increased in the CST among the iNPH patients, although such increase was not observed in the CC of these patients. The authors reported that the observed gait abnormalities among the patients with iNPH were statistically significantly correlated with the DTI results in the CST: they noted a strong positive correlation $(\mathrm{p}<0.001)$ between MD and the number of steps and walking duration. ${ }^{14}$ In addition, FA in the CST was weakly $(p=0.04)$ and negatively correlated with both of these gait variables.

In addition, Hattori et al. used TBSS, ROI, and tractspecific analysis methods to assess FA in the CST in 20 patients with iNPH and in 20 age-matched, healthy controls. ${ }^{15}$ Fractional anisotropy in the CST showed no patterns specific to iNPH. White-matter abnormalities vary in iNPH patients, an observation that was also confirmed by significantly decreased FA in the WM located in the frontal and the parietal lobes and by significantly increased FA in the IC..$^{15}$

Similarly, Koyama et al. used DTI metrics in $24 \mathrm{iNPH}$ patients and 21 healthy controls to generate FA brain maps and determine TBSS to measure FA in selected ROIs (the $\mathrm{CC}$, posterior limb of the internal capsule [IC], and cerebral peduncles)..$^{29}$ Compared with the controls, patients with iNPH had significantly lower FA in the CC and significantly higher FA in the posterior limb of the IC. The authors also reported that gait abnormalities of the iNPH patients were statistically significantly correlated with the lower FA in the CC. ${ }^{29}$

In another study, Koyama et al., studying 10 patients with iNPH and 10 age-matched controls, ${ }^{30}$ used a computer-automated method to generate FA brain maps adjusted to the standard-stereotactic space. The authors focused on 2 ROIs: the minor forceps and the CST. In both of these regions, FA was decreased among the patients with iNPH, but this decrease did not reach statistical significance. Moreover, the authors postulated that the FA in the minor forceps was negatively correlated with the clinical manifestations of iNPH such as urinary incontinence and gait disturbances. ${ }^{30}$ Similarly, in a study of 11 iNPH patients and 6 healthy controls, Nakanishi et al. used tract-specific analysis, mean diffusional kurtosis imaging (DKI), and DTI of the CST at the level of the lateral ventricle and observed that DKI and axial diffusion kurtosis values were significantly lower in the iNPH patients than in the controls. ${ }^{42}$ In contrast, ADCs, FA, and axial (i.e., $\lambda_{1}$ ) eigenvalues were significantly higher in the patients with iNPH.

Recently, Osawa and colleagues, studying 6 iNPH patients and 12 healthy controls, used a new metric, the delta-ADC, which determines the changes in ADCs in the brain during one cardiac cycle. ${ }^{45}$ Delta-ADCs in the frontal, temporal, and occipital lobes were significantly higher in the iNPH patients than in the control patients. Although ADCs were also significantly higher in the iNPH group than the control group, these differences were smaller than those observed in the delta-ADCs. The authors also reported that ADCs and delta-ADCs were not significantly correlated with each other. ${ }^{45}$

\section{DTI Characteristics in iNPH and Other Neurodegenerative Diseases With Similar Signs and Symptoms}

Daouk et al. studied DTI patterns in WM in the region of the IC, ventricular morphometry, and CSF flow in patients with iNPH or AD.? The authors reported that FA was statistically significantly correlated with the ventricular dimensions in the patients with iNPH $(\mathrm{p}<0.001$, Spearman's rho $=0.88$ ). In the patients with $\mathrm{AD}, \mathrm{CSF}$ aqueductal flow was statistically significantly correlated with the ADC ( $p<0.001$, Spearman's rho = 0.79). Although the authors noted abnormalities in the CSF flow in both patient groups, patients with iNPH appeared to have different DTI patterns in the region of the IC, which could be a significant imaging finding that could differentiate these 2 pathological entities?

Hattori and colleagues investigated the differences in DTI findings among 18 patients with iNPH, 11 patients with $\mathrm{AD}, 11$ patients with $\mathrm{PD}$ and dementia, and 19 healthy controls. ${ }^{17}$ The authors used DTI of the CST to measure FA and axial and radial eigenvalues with a tractspecific analysis. Only FA and axial eigenvalues were significantly higher in the iNPH group than in the other 3 groups $(\mathrm{p}<0.001)$. The CST tractography in patients with iNPH revealed a qualitative pattern that was different from the patterns in the other patient groups, and this difference was statistically significant $(\mathrm{p}<0.001)$. The authors reported that the analysis of FA in the CST had 94\% sensitivity and $80 \%$ specificity in detecting iNPH. ${ }^{17}$

In a further study, Hattori et al., using tract-specific analysis, performed DTI of the fornix in 22 patients with iNPH, 20 patients with AD, and 20 healthy controls. ${ }^{16}$ In addition, the authors recorded the volume, length, and mean cross-sectional area of the fornix in the study participants. The iNPH and AD groups had a significantly smaller volume of the fornix and had significantly lower FA and smaller cross-sectional areas in this region than the healthy controls. Interestingly, the fornix was significantly longer in iNPH patients than in the AD patients and healthy controls. ${ }^{16}$

Hong et al. performed DTI of the 3 main regions of the hippocampus (i.e., the head, body, and tail) in $13 \mathrm{iNPH}$ patients, $15 \mathrm{AD}$ patients, and 15 healthy controls. ${ }^{18}$ The authors measured DTI variables in both hemispheres and used the ROI-selection technique. They reported that the tissue volume in the hippocampus did not significantly differ between the iNPH and AD groups. Fractional anisotropy was lowest in the AD patients, whereas MD was the highest in these patients. For the patients with iNPH, the FA and MD values were in between the 3 groups. Healthy controls had the highest FA and the lowest MD of the 3 groups investigated. The authors also noted that ROI or laterality of the hippocampus has insignificant influence on FA and MD results. ${ }^{18}$

Ivkovic and coworkers employed a new DTI technique based on 3T MRI sequences in 15 patients with possible iNPH; 25 patients with various disorders, including AD, $\mathrm{PD}$, or Lewy body dementia; and 2 patients with both NPH and $\mathrm{AD} .{ }^{20}$ The authors used MD histograms to discriminate the brain and the ventricles from a theoretical third compartment consisting mostly of partial-volume voxels and of voxels with high MD determined with a combina- 
tion of DTI and diffusion-weighted imaging techniques. The authors' novel technique efficiently recognized and distinguished the 3 pathological conditions with $86 \%$ sensitivity and $96 \%$ specificity, respectively. However, the specificity of this new method dropped to $88 \%$ when it was used to discriminate patients with iNPH from those with $\mathrm{AD}{ }^{20}$

Kanno et al. used the DTI technique to determine MD and FA from a voxel-based statistical map to distinguish iNPH (in 20 patients) from AD (20 patients) and PD (20 patients). ${ }^{24}$ In the supratentorial WM areas, the highest MD and the lowest FA were observed in the patients with iNPH. The periventricular areas exhibited high MD, while the $\mathrm{CC}$ and subcortical areas had low FA in the patients with iNPH. In addition, only the mean hemispherical FA values were partially associated with the clinical severity of iNPH. The authors also reported that the FA results obtained from the anterior limb of the IC and from the region under the left supplementary motor area were associated with more severed gait abnormalities in the patients with iNPH. Using the frontal assessment battery method, an evaluation tool that evaluates the functionality of the frontal lobes and reveals cognitive impairment in patients with dementia, ${ }^{12}$ Kanno and coworkers also observed that FA in the frontal and the parietal subcortical WM was statistically significantly correlated with frontal lobe functionality in iNPH patients. ${ }^{24}$

Marumoto and others investigated FA in 5 different brain regions in $10 \mathrm{iNPH}$ patients, 18 PD patients, and 10 healthy controls. ${ }^{37}$ According to this study, FA in the anterior thalamic radiation and the minor forceps was lower in iNPH patients than in the PD patients. Moreover, FA in the anterior thalamic radiation was statistically significantly associated with gait disturbances in both patient groups. ${ }^{37}$

\section{DTI Characteristics in iNPH Patients Undergoing CSF Drainage or Shunt Placement}

Demura et al. determined FA and ADCs in several WM regions in 36 patients with possible iNPH before and 24 hours after a CSF tap test. ${ }^{9}$ The patients were separated into 2 groups: those who had significant neurological improvement after the CSF tap test (17 patients) and those who had no improvement (19 patients). A statistically significant decrease $(\mathrm{p}<0.05)$ in ADCs was detected only for the patients with neurological improvement. In contrast, a significant increase $(\mathrm{p}<0.05)$ in FA after the CSF tap test was observed in both groups. ${ }^{9}$

Jurcoane et al. studied 2 groups of patients with iNPH after they had undergone CSF drainage via a spinal tap: the first group comprised 12 patients whose gait significantly improved 3 days after the CSF drainage, and the second group consisted of 14 patients whose gait did not improve. ${ }^{22}$ The authors performed DTI and magnetization transfer ratio (MTR) measurements in the CSTs after the CSF drainage in both patient groups. In addition, the DTI and MTR assessments were repeated in the patients of the first group after undergoing ventriculoperitoneal shunt placement. As a reference, the authors used the DTI and MTR results in the superior longitudinal fasciculus before and after CSF removal in the iNPH groups and also from 10 age-matched, healthy controls. The study participants in the control group had statistically significantly lower $(\mathrm{p}<0.001)$ axial diffusivity than the patients in the $2 \mathrm{iNPH}$ groups. In addition, the patients whose gait had improved after CSF drainage had higher FA than the healthy controls, and both axial diffusivity and FA statistically significantly decreased after the shunt placement in these patients. The authors concluded that a reduction in axial diffusivity of greater than $0.7 \%$ could accurately discriminate with $82 \%$ sensitivity between those iNPH patients who respond to CSF drainage and those who do not. The authors also postulated that when the axial diffusivity decrease reaches $1 \%$, it could accurately predict with $87.5 \%$ sensitivity and $75.0 \%$ specificity which iNPH patients would show overall clinical improvement after shunt placement. They also concluded that using only DTI measurements before CSF drainage in their study could not differentiate those responding to the drainage from those who do not. ${ }^{22}$

Lenfeldt et al. performed DTI in 18 iNPH patients before and 3 days after removal of 400 milliliters CSF via a spinal tap and also in 10 healthy controls. ${ }^{34}$ The authors reported that FA in the anterior frontal WM was significantly lower in the iNPH patients than in the controls. In addition, the ADCs in the genu of the $\mathrm{CC}$ and the centrum semiovale were elevated in the iNPH patients compared with the ADCs in the healthy controls. The authors also noted that in all iNPH patients, the ADCs did not change after the CSF drainage. ${ }^{34}$

In a study of 24 patients with clinical and imaging manifestations of iNPH and 14 healthy controls, ReissZimmermann et al. studied DTI changes in the patients with iNPH after CSF drainage. ${ }^{46}$ Of note, only 15 of the patients were eventually included in the authors' study protocol. All DTI measurements (i.e., FA and mean, parallel, and radial diffusivities) were performed before and after (within 6-36 hours) lumbar drainage. The results of this study indicated that FA was higher in the subcortical WM and the CC in patients with iNPH than in the controls. In addition, the increased radial diffusivity tended to normalize after the CSF drainage. ${ }^{46}$

Kim et al. employed DTI in 16 patients with iNPH before surgery and also in 10 patients with $\mathrm{AD}, 10$ patients with subcortical vascular dementia, and 20 healthy controls. ${ }^{27}$ The imaging protocol was repeated in $9 \mathrm{iNPH}$ patients who had undergone CSF shunt placement. The authors reported that the presurgical iNPH group had the highest FA of all groups in the posterior limb of the IC. They also observed that FA significantly decreased after the shunt placement. Before the shunting, the iNPH patients had MD values in the anterior periventricular WM, the anterior limb of the IC, and the superior longitudinal fasciculus that were higher than in the patients with $\mathrm{AD}$ and in the healthy controls but were lower than in the patients with subcortical vascular dementia. The authors also reported that FA could diagnose iNPH with $87.5 \%$ sensitivity and $95.0 \%$ specificity. ${ }^{27}$

Using a new 12-direction sequence acquisition with a 3T MRI unit, Nicot et al. investigated alterations in FA and also in the ADC in the IC and the body of the CC in $10 \mathrm{iNPH}$ patients after the patients had undergone a shunt placement. ${ }^{43}$ The authors' measurements, obtained with a 
conventional DTI protocol, showed that only the ADCs in the IC were significantly higher postoperatively. In contrast, the novel image acquisition technique indicated a significant postoperative decrease in FA.

\section{Discussion}

Several hypotheses have been proposed for explaining the underlying pathophysiological mechanisms of iNPH. Most of these theories converge on the concept that ventricular enlargement stretches and compresses the periventricular WM and the adjacent CC, leading to interstitial edema and progressive axonal loss. ${ }^{2,3,11,32,38,48,55}$ Neuropathological findings in NPH are consistent with demyelination of the frontal lobes, presence of microinfarctions, organized thrombi or lacunas, arteriole sclerosis of the periventricular WM, and leptomeningeal fibrosis. ${ }^{5,10} \mathrm{Com}-$ pression or deformation of the CST due to the ventricular enlargement results in frontal lobe dysfunction, malfunction of the corticobasal ganglia-thalamic circuit, or both. This malfunction greatly affects the supplementary motor cortex, resulting in the gait disturbances characteristic of patients with NPH. ${ }^{33,40,44,47}$ Numerous similarities in pathogenesis between iNPH and AD or other subcortical vascular dementias have been reported. ${ }^{6,23,52-54}$ Symptoms of iNPH resemble those observed in disorders arising from diseases of the frontal lobe. ${ }^{51,56}$ Biopsy samples taken at the time of shunt placement in patients with iNPH have shown the presence of AD-type histological changes, which may explain the lack of treatment responses after shunt placements in these cases. ${ }^{8,49,50}$

Several researchers have underlined the significance of DTI-based methods for diagnosing iNPH. ${ }^{14,15,29,30,42,45} \mathrm{~A}$ plethora of different DTI protocols for examining various areas of the brain have been described in the literature, including manual ROI placement, TBSS, tract-specific, voxel-based, and delta-ADC analyses. The most frequently studied regions in patients with iNPH are the periventricular WM areas such as the IC, CST, and CC, as well as the frontal, temporal, and occipital lobes and the cerebral peduncles. Patients with iNPH have significantly higher MD in the CST and the CC than do healthy controls. ${ }^{14} \mathrm{In}$ addition, most studies have reported significantly higher FA in the CST among iNPH patients than among healthy subjects..$^{15,42}$ The only exception was the study by Koyama et al., which reported decreased FA values in the CST and the minor forceps in iNPH patients. ${ }^{30}$ Significantly less experience exists with the use of DKI for evaluating or diagnosing iNPH. It has been reported, however, that DKI values in the CST are significantly decreased in iNPH patients compared with those in healthy controls. ${ }^{42}$ The delta-ADC has also been used for examining patients with suspected iNPH, and it has been reported that delta-ADCs in the frontal, temporal, and occipital lobes are increased in iNPH patients relative to the corresponding delta-ADCs in healthy controls..$^{45}$ Furthermore, several clinical investigators have reported that the gait abnormalities of iNPH patients are statistically significantly correlated with increased FA in the CST and the minor forceps..$^{14,30,37}$

A critical analysis of all published series employing DTI with combined FA and MD measurements in CNS areas such as the CST and the CC may help improve the diagnosis of NPH. Additionally, FA and MD both seem to correlate well with NPH symptoms, particularly with gait disturbance. Thus, the DTI method may be a useful noninvasive diagnostic tool that not only can help diagnose NPH, but may also assist in the grading of the clinical severity and progression of NPH. We note that the number of published series on DTI for NPH diagnosis is still small and that these studies had relatively low numbers of participants. In addition, some normal, age-related changes in brain function and structure in these participants may have also influenced the accuracy of this imaging method. Despite these limiting factors, the available data support the inclusion of DTI in preoperative imaging assessments during the examination of patients with suspected NPH.

In addition, FA and MD may well be important for differentiating iNPH from other neurodegenerative diseases such as AD and PD that frequently have overlapping clinical symptoms and signs. ${ }^{7,16-18,20,27,37}$ It has been shown that iNPH and AD patients have different DTI findings in the IC. ${ }^{7}$ Moreover, it also has been reported that the ventricular volume is positively correlated with FA in patients with iNPH. ${ }^{7}$ Characteristically, FA in various periventricular areas in patients with iNPH statistically significantly differs from that in patients with PD or AD. For example, FA and axial eigenvalues in the CST were reported to be significantly higher in patients with iNPH than in PD or AD patients. ${ }^{17}$ Of note, FA has a sensitivity of $94 \%$ and a specificity of $80 \%$ for diagnosing iNPH. ${ }^{17}$ In addition, FA in the fornix is significantly lower in patients with iNPH than in those with AD. ${ }^{16}$ Patients with iNPH have higher FA in the hippocampus than do AD patients, but lower FA in the same region than healthy controls. ${ }^{18}$ Moreover, MD in the hippocampus in iNPH patients was reported to be significantly lower than in AD patients but significantly higher than in healthy controls..$^{18}$ Similarly, FA values in the posterior limb of the IC and MD in the anterior limb of the IC and in the superior longitudinal fasciculus are significantly higher in patients with iNPH than in patients with AD. ${ }^{27}$ Also, iNPH patients have lower FA in the anterior thalamic radiation and the minor forceps than do PD patients..$^{37}$ The use of the MD histogram method appears to have had a significant role in differentiating iNPH from AD, PD, or Lewy body dementia. ${ }^{20}$ This MD method has been reported to have $86 \%$ sensitivity and $96 \%$ specificity in differentiating iNPH from the other neurodegenerative diseases studied. ${ }^{20}$

Differentiating iNPH from other neurodegenerative diseases represents a frequent and complex diagnostic problem, which can be addressed with the use of DTI metrics. Although previously published morphometric changes in the fornix and the hippocampus appear to be contradictory, their evaluation along with DTI findings may help clinicians differentiate iNPH from other neurodegenerative diseases. Newer techniques for analyzing $\mathrm{MD}$ and FA data may provide noninvasive tools for differential diagnosis with high specificity and sensitivity. Again, the limiting factors of a small numbers of series and study sizes decrease the statistical significance of the impact of these new imaging-based methods on improved iNPH diagnosis. 
Moreover, the utility of DTI with FA and ADC techniques has been studied also in patients with iNPH before and after CSF drainage via a spinal tap to identify imaging characteristics that could be used to predict which patients would most likely benefit from the drainage., $, 22,27,34$, ${ }^{43,46}$ Some of these studies have reported that CSF drainage significantly increases FA in the periventricular WM and the CC and also significantly decreases ADC in these areas. ${ }^{9,46}$ The authors observed that elevated radial diffusivity in the subcortical WM returned to normal after CSF drainage in iNPH patients with clinical improvement. ${ }^{9,46}$ These findings may have a predictive value in selecting those patients whose NPH might respond to a CSF shunt placement. However, 1 study reported that the DTI variables did not change after CSF drainage. ${ }^{34}$

In addition, previous series have investigated the utility of DTI also in patients undergoing CSF shunt procedures, and all have noted that FA values in the CST and the IC decrease after shunt placement. ${ }^{22,27,43}$ Moreover, shunt placement increases ADC. ${ }^{43}$ It has also been proposed that the significant decrease in axial diffusivity after shunting may have a predictive value for selecting patient with iNPH for surgery. ${ }^{22}$ In light of these findings, we emphasize the importance of adding DTI as a noninvasive diagnostic tool to our armamentarium to identify iNPH patients who would respond to a CSF shunt placement.

\section{Conclusions}

Although DTI is a relatively new imaging modality, it may take on a significant role in the diagnosis and differentiation of iNPH from other disorders and diseases with similar imaging and clinical features. The reported specificities and sensitivities of this noninvasive imaging method for detecting iNPH are high. Moreover, the use of DTI could significantly contribute to the selection of iNPH patients for surgery, potentially improving overall outcomes for these patients and mitigating surgical failure rates.

However, we wish to highlight significant drawbacks to the wide clinical application of the DTI method. First, the existence of various technical protocols, along with the significant variation in the anatomical areas studied, greatly decreases the reproducibility and diagnostic accuracy of both FA and MD. The use of these different techniques and metrics makes a meaningful comparison of previously published results practically impossible. Second, the normal range of the parameters measured needs to be defined and most probably needs to be standardized for different age groups. This will require large-scale, multicenter studies and the adoption of a widely accepted imaging protocol for creating a database and for setting normal measurement ranges. Despite these technical limitations and weaknesses, DTI lends itself to the examination of iNPH patients and may well improve overall outcomes for these patients.

\section{References}

1. Adams RD, Fisher CM, Hakim S, Ojemann RG, Sweet WH: Symptomatic occult hydrocephalus with "normal" cerebrospinal-fluid pressure-a treatable syndrome. N Engl J Med 273:117-126, 1965
2. Akai K, Uchigasaki S, Tanaka U, Komatsu A: Normal pressure hydrocephalus. Neuropathological study. Acta Pathol Jpn 37:97-110, 1987

3. Anik Y, Demirci A, Anik I, Etus V, Arslan A: Apparent diffusion coefficient and cerebrospinal fluid flow measurements in patients with hydrocephalus. J Comput Assist Tomogr 32:392-396, 2008

4. Basser PJ, Jones DK: Diffusion-tensor MRI: theory, experimental design and data analysis - a technical review. NMR Biomed 15:456-467, 2002

5. Bech RA, Juhler M, Waldemar G, Klinken L, Gjerris F: Frontal brain and leptomeningeal biopsy specimens correlated with cerebrospinal fluid outflow resistance and B-wave activity in patients suspected of normal-pressure hydrocephalus. Neurosurgery 40:497-502, 1997

6. Curran T, Lang AE: Parkinsonian syndromes associated with hydrocephalus: case reports, a review of the literature, and pathophysiological hypotheses. Mov Disord 9:508-520, 1994

7. Daouk J, Chaarani B, Zmudka J, Capel C, Fichten A, Bouzerar R, et al: Relationship between cerebrospinal fluid flow, ventricles morphology, and DTI properties in internal capsules: differences between Alzheimer's disease and normalpressure hydrocephalus. Acta Radiol 55:992-999, 2014

8. Del Bigio MR, Cardoso ER, Halliday WC: Neuropathological changes in chronic adult hydrocephalus: cortical biopsies and autopsy findings. Can J Neurol Sci 24:121-126, 1997

9. Demura K, Mase M, Miyati T, Osawa T, Hattori M, Kasai H, et al: Changes of fractional anisotropy and apparent diffusion coefficient in patients with idiopathic normal pressure hydrocephalus. Acta Neurochir Suppl 113:29-32, 2012

10. Deo-Narine V, Gomez DG, Vullo T, Manzo RP, Zimmerman $\mathrm{RD}$, Deck MD, et al: Direct in vivo observation of transventricular absorption in the hydrocephalic dog using magnetic resonance imaging. Invest Radiol 29:287-293, 1994

11. Ding Y, McAllister JP II, Yao B, Yan N, Canady AI: Axonal damage associated with enlargement of ventricles during hydrocephalus: a silver impregnation study. Neurol Res 23:581-587, 2001

12. Dubois B, Slachevsky A, Litvan I, Pillon B: The FAB: A Frontal Assessment Battery at bedside. Neurology 55:16211626,2000

13. Hakim S, Adams RD: The special clinical problem of symptomatic hydrocephalus with normal cerebrospinal fluid pressure. Observations on cerebrospinal fluid hydrodynamics. J Neurol Sci 2:307-327, 1965

14. Hattingen E, Jurcoane A, Melber J, Blasel S, Zanella FE, Neumann-Haefelin T, et al: Diffusion tensor imaging in patients with adult chronic idiopathic hydrocephalus. Neurosurgery 66:917-924, 2010

15. Hattori T, Ito K, Aoki S, Yuasa T, Sato R, Ishikawa M, et al: White matter alteration in idiopathic normal pressure hydrocephalus: tract-based spatial statistics study. AJNR Am J Neuroradiol 33:97-103, 2012

16. Hattori T, Sato R, Aoki S, Yuasa T, Mizusawa H: Different patterns of fornix damage in idiopathic normal pressure hydrocephalus and Alzheimer disease. AJNR Am J Neuroradiol 33:274-279, 2012

17. Hattori T, Yuasa T, Aoki S, Sato R, Sawaura H, Mori T, et al: Altered microstructure in corticospinal tract in idiopathic normal pressure hydrocephalus: comparison with Alzheimer disease and Parkinson disease with dementia. AJNR Am J Neuroradiol 32:1681-1687, 2011

18. Hong YJ, Yoon B, Shim YS, Cho AH, Lim SC, Ahn KJ, et al: Differences in microstructural alterations of the hippocampus in Alzheimer disease and idiopathic normal pressure hydrocephalus: a diffusion tensor imaging study. AJNR Am J Neuroradiol 31:1867-1872, 2010

19. Horsfield MA, Jones DK: Applications of diffusion-weighted 
and diffusion tensor MRI to white matter diseases-a review. NMR Biomed 15:570-577, 2002

20. Ivkovic M, Liu B, Ahmed F, Moore D, Huang C, Raj A, et al: Differential diagnosis of normal pressure hydrocephalus by MRI mean diffusivity histogram analysis. AJNR Am J Neuroradiol 34:1168-1174, 2013

21. Jaraj D, Agerskov S, Rabiei K, Marlow T, Jensen C, Guo X, et al: Vascular factors in suspected normal pressure hydrocephalus: a population-based study. Neurology 86:592-599, 2016

22. Jurcoane A, Keil F, Szelenyi A, Pfeilschifter W, Singer OC, Hattingen E: Directional diffusion of corticospinal tract supports therapy decisions in idiopathic normal-pressure hydrocephalus. Neuroradiology 56:5-13, 2014

23. Kalaria RN, Erkinjuntti T: Small vessel disease and subcortical vascular dementia. J Clin Neurol 2:1-11, 2006

24. Kanno S, Abe N, Saito M, Takagi M, Nishio Y, Hayashi A, et al: White matter involvement in idiopathic normal pressure hydrocephalus: a voxel-based diffusion tensor imaging study. J Neurol 258:1949-1957, 2011

25. Kapsalaki E, Svolos P, Tsougos I, Theodorou K, Fezoulidis I, Fountas KN: Quantification of normal CSF flow through the aqueduct using PC-cine MRI at 3T. Acta Neurochir Suppl 113:39-42, 2012

26. Kazui H: [Current state of diagnosis and treatment of idiopathic normal pressure hydrocephalus.] Brain Nerve 68:429-440, 2016 (Jpn)

27. Kim MJ, Seo SW, Lee KM, Kim ST, Lee JI, Nam DH, et al: Differential diagnosis of idiopathic normal pressure hydrocephalus from other dementias using diffusion tensor imaging. AJNR Am J Neuroradiol 32:1496-1503, 2011

28. Klinge PM, Brooks DJ, Samii A, Weckesser E, van den Hoff J, Fricke H, et al: Correlates of local cerebral blood flow $(\mathrm{CBF})$ in normal pressure hydrocephalus patients before and after shunting-a retrospective analysis of $\left[{ }^{15} \mathrm{O}\right] \mathrm{H}_{2} \mathrm{O}$ PET-CBF studies in 65 patients. Clin Neurol Neurosurg 110:369-375, 2008

29. Koyama T, Marumoto K, Domen K, Miyake H: White matter characteristics of idiopathic normal pressure hydrocephalus: a diffusion tensor tract-based spatial statistic study. Neurol Med Chir (Tokyo) 53:601-608, 2013

30. Koyama T, Marumoto K, Domen K, Ohmura T, Miyake H: Diffusion tensor imaging of idiopathic normal pressure hydrocephalus: a voxel-based fractional anisotropy study. Neurol Med Chir (Tokyo) 52:68-74, 2012

31. Larsson A, Arlig A, Bergh AC, Bilting M, Jacobsson L, Stephensen H, et al: Quantitative SPECT cisternography in normal pressure hydrocephalus. Acta Neurol Scand 90:190196, 1994

32. Leliefeld PH, Gooskens RH, Braun KP, Ramos LM, Uiterwaal CS, Regli LP, et al: Longitudinal diffusion-weighted imaging in infants with hydrocephalus: decrease in tissue water diffusion after cerebrospinal fluid diversion. J Neurosurg Pediatr 4:56-63, 2009

33. Lenfeldt N, Larsson A, Nyberg L, Andersson M, Birgander $\mathrm{R}$, Eklund A, et al: Idiopathic normal pressure hydrocephalus: increased supplementary motor activity accounts for improvement after CSF drainage. Brain 131:2904-2912, 2008

34. Lenfeldt N, Larsson A, Nyberg L, Birgander R, Eklund A, Malm J: Diffusion tensor imaging reveals supplementary lesions to frontal white matter in idiopathic normal pressure hydrocephalus. Neurosurgery 68:1586-1593, 2011

35. Malloy P, Correia S, Stebbins G, Laidlaw DH: Neuroimaging of white matter in aging and dementia. Clin Neuropsychol 21:73-109, 2007

36. Malm J, Kristensen B, Stegmayr B, Fagerlund M, Koskinen LO: Three-year survival and functional outcome of patients with idiopathic adult hydrocephalus syndrome. Neurology 55:576-578, 2000
37. Marumoto K, Koyama T, Hosomi M, Kodama N, Miyake H, Domen K: Diffusion tensor imaging in elderly patients with idiopathic normal pressure hydrocephalus or Parkinson's disease: diagnosis of gait abnormalities. Fluids Barriers CNS 9:20, 2012

38. Mataró M, Matarín M, Poca MA, Pueyo R, Sahuquillo J, Barrios M, et al: Functional and magnetic resonance imaging correlates of corpus callosum in normal pressure hydrocephalus before and after shunting. J Neurol Neurosurg Psychiatry 78:395-398, 2007

39. McAllister JP II, Williams MA, Walker ML, Kestle JR, Relkin NR, Anderson AM, et al: An update on research priorities in hydrocephalus: overview of the third National Institutes of Health-sponsored symposium "Opportunities for Hydrocephalus Research: Pathways to Better Outcomes.” J Neurosurg 123:1427-1438, 2015

40. Miyoshi N, Kazui H, Ogino A, Ishikawa M, Miyake H, Tokunaga $\mathrm{H}$, et al: Association between cognitive impairment and gait disturbance in patients with idiopathic normal pressure hydrocephalus. Dement Geriatr Cogn Disord 20:71-76, 2005

41. Momjian S, Owler BK, Czosnyka Z, Czosnyka M, Pena A, Pickard JD: Pattern of white matter regional cerebral blood flow and autoregulation in normal pressure hydrocephalus. Brain 127:965-972, 2004

42. Nakanishi A, Fukunaga I, Hori M, Masutani Y, Takaaki H, Miyajima M, et al: Microstructural changes of the corticospinal tract in idiopathic normal pressure hydrocephalus: a comparison of diffusion tensor and diffusional kurtosis imaging. Neuroradiology 55:971-976, 2013

43. Nicot B, Bouzerar R, Gondry-Jouet C, Capel C, Peltier J, Fichten A, et al: Effect of surgery on periventricular white matter in normal pressure hydrocephalus patients: comparison of two methods of DTI analysis. Acta Radiol 55:614621,2014

44. Nowak DA, Gumprecht H, Topka H: CSF drainage ameliorates the motor deficit in normal pressure hydrocephalus: evidence from the analysis of grasping movements. J Neurol 253:640-647, 2006

45. Osawa T, Mase M, Miyati T, Kan H, Demura K, Kasai H, et al: Delta-ADC (apparent diffusion coefficient) analysis in patients with idiopathic normal pressure hydrocephalus. Acta Neurochir Suppl 114:197-200, 2012

46. Reiss-Zimmermann M, Scheel M, Dengl M, Preuß M, Fritzsch D, Hoffmann KT: The influence of lumbar spinal drainage on diffusion parameters in patients with suspected normal pressure hydrocephalus using 3T MRI. Acta Radiol 55:622-630, 2014

47. Robbins TW, James M, Owen AM, Lange KW, Lees AJ, Leigh PN, et al: Cognitive deficits in progressive supranuclear palsy, Parkinson's disease, and multiple system atrophy in tests sensitive to frontal lobe dysfunction. J Neurol Neurosurg Psychiatry 57:79-88, 1994

48. Röricht S, Meyer BU, Woiciechowsky C, Lehmann R: Callosal and corticospinal tract function in patients with hydrocephalus: a morphometric and transcranial magnetic stimulation study. J Neurol 245:280-288, 1998

49. Savolainen S, Laakso MP, Paljärvi L, Alafuzoff I, Hurskainen $\mathrm{H}$, Partanen K, et al: MR imaging of the hippocampus in normal pressure hydrocephalus: correlations with cortical Alzheimer's disease confirmed by pathologic analysis. AJNR Am J Neuroradiol 21:409-414, 2000

50. Savolainen S, Paljärvi L, Vapalahti M: Prevalence of Alzheimer's disease in patients investigated for presumed normal pressure hydrocephalus: a clinical and neuropathological study. Acta Neurochir (Wien) 141:849-853, 1999

51. Shprecher D, Schwalb J, Kurlan R: Normal pressure hydrocephalus: diagnosis and treatment. Curr Neurol Neurosci Rep 8:371-376, 2008 
52. Silverberg GD, Mayo M, Saul T, Rubenstein E, McGuire D: Alzheimer's disease, normal-pressure hydrocephalus, and senescent changes in CSF circulatory physiology: a hypothesis. Lancet Neurol 2:506-511, 2003

53. Stolze H, Kuhtz-Buschbeck JP, Drücke H, Jöhnk K, Illert M, Deuschl G: Comparative analysis of the gait disorder of normal pressure hydrocephalus and Parkinson's disease. J Neurol Neurosurg Psychiatry 70:289-297, 2001

54. Tullberg M, Hultin L, Ekholm S, Månsson JE, Fredman P, Wikkels $\varnothing \mathrm{C}$ : White matter changes in normal pressure hydrocephalus and Binswanger disease: specificity, predictive value and correlations to axonal degeneration and demyelination. Acta Neurol Scand 105:417-426, 2002

55. Uluğ AM, Truong TN, Filippi CG, Chun T, Lee JK, Yang C, et al: Diffusion imaging in obstructive hydrocephalus. AJNR Am J Neuroradiol 24:1171-1176, 2003

56. Walchenbach R, Geiger E, Thomeer RT, Vanneste JA: The value of temporary external lumbar CSF drainage in predicting the outcome of shunting on normal pressure hydrocephalus. J Neurol Neurosurg Psychiatry 72:503-506, 2002

57. Waldemar G, Schmidt JF, Delecluse F, Andersen AR, Gjerris F, Paulson OB: High resolution SPECT with [99mTc]-d,1-HM$\mathrm{PAO}$ in normal pressure hydrocephalus before and after shunt operation. J Neurol Neurosurg Psychiatry 56:655-664, 1993
58. Williams MA, Malm J: Diagnosis and treatment of idiopathic normal pressure hydrocephalus. Continuum (Minneap Minn) 22 (2 Dementia):579-599, 2016

\section{Disclosures}

The authors report no conflict of interest concerning the materials or methods used in this study or the findings specified in this paper.

\section{Author Contributions}

Conception and design: Siasios, Fountas. Acquisition of data: Siasios, Fotiadou. Analysis and interpretation of data: Siasios, Kapsalaki, Fountas. Drafting the article: Siasios, Kapsalaki, Fountas, Fotiadou. Critically revising the article: Dimopoulos, Siasios, Kapsalaki, Fountas, Dorsch, Vakharia, Pollina. Reviewed submitted version of manuscript: all authors.

\section{Correspondence}

Vassilios G. Dimopoulos, Department of Neurosurgery, University at Buffalo, 100 High St., Ste. B4, Buffalo, NY 14203. email: vdimopoulos@ubns.com. 